\title{
THE SECOND WEIGHTED MOMENT OF $\zeta$
}

\author{
by
}

S. Bettin \& J.B. Conrey

\begin{abstract}
We give an explicit formula for the second weighted moment of $\zeta(s)$ on the critical line tailored for fast computations with any desired accuracy.

Résumé. - Nous donnons une formule explicite pour le second moment pondéré de la fonction $\zeta$ sur la droite critique permettant une évaluation rapide pour n'importe quel degré de précision.
\end{abstract}

\section{Introduction}

For $\Im z>0$ let

$$
E_{1}(z)=1-4 \sum_{n=1}^{\infty} d(n) e(n z)
$$

and let

$$
E_{1}(z)-(1 / z) E_{1}(-1 / z)=: \psi(z) .
$$

Then $\psi$ is analytic in $\mathbb{C}^{\prime}$, the complex plane minus the negative real-axis and is given in that region by

$$
\psi(z)=-2 \frac{\log (2 \pi z)-\gamma}{\pi i z}-\frac{2}{\pi} \int_{(-1 / 2)} \frac{\zeta(s) \zeta(1-s)}{\sin \pi s} z^{-s} d s .
$$

Moreover, $\psi$ satisfies a 3-term relation

$$
\psi(z+1)=\psi(z)-\frac{1}{z+1} \psi\left(\frac{z}{z+1}\right)
$$

2010 Mathematics Subject Classification. - 11M06.

Key words and phrases. - Riemann zeta function, Moments.

Research supported in part by a grant from the National Science Foundation. 
and for $|z| \leq 1$ satisfies

$$
\frac{\pi i}{2} \psi(1+z)=\frac{-\log (1+z)-1}{1+z}+\frac{1}{1+z} \sum_{m=1}^{\infty} a_{m}(-1)^{m} z^{m}
$$

where, for $m \geq 1$,

$$
a_{m}=\frac{-1}{m+1}+2 b_{m}+2 \sum_{j=0}^{m-2}\left(\begin{array}{c}
m-1 \\
j
\end{array}\right) b_{j+2}
$$

with $b_{1}=0$ and

$$
b_{n}=\frac{\zeta(n) B_{n}}{n}
$$

for $n \geq 2$. The $a_{m}$ are extremely small:

$$
a_{m}=2^{5 / 4} \pi^{3 / 4} \frac{e^{-2 \sqrt{\pi m}}}{m^{3 / 4}}\left(\sin \left(2 \sqrt{\pi m}+\frac{3}{8} \pi\right)+O\left(\frac{1}{\sqrt{m}}\right)\right)
$$

so that the series $\sum a_{m} z^{m}$ converges everywhere on its circle of convergence $|z|=1$. All of these results can be found in $[\mathbf{B C}]$.

\section{The second moment of $\zeta$}

Theorem 1. - Let

$$
I(\delta)=\int_{0}^{\infty}|\zeta(1 / 2+i t)|^{2} e^{-\delta t} d t .
$$

Then, for $0<\Re(\delta)<\pi$ and $\Im \delta \leq 0$ we have

$$
I(\delta)=C_{0}(\delta)+C_{1}(\delta)+C_{2}(\delta)+C_{3}(\delta)
$$

where

$$
\begin{gathered}
C_{0}(\delta)=-\frac{\left(\log \left(1-e^{-i \delta}\right)+1\right)}{2 \sin \frac{\delta}{2}}+\frac{\frac{\pi}{2} e^{-i \delta / 2}}{1-e^{i \delta}}+e^{i \delta / 2}(-\delta+\pi / 2+i \gamma-i \log 2 \pi) \\
C_{1}(\delta)=\frac{1}{2 \sin \frac{\delta}{2}} \sum_{m=1}^{\infty} a_{m} e^{-i m \delta}
\end{gathered}
$$

where $a_{m}=\frac{-1}{m+1}+2 b_{m}+2 \sum_{j=0}^{m-2}\left(\begin{array}{c}m-1 \\ j\end{array}\right) b_{j+2}$ with $b_{1}=0$ and $b_{n}=\frac{\zeta(n) B_{n}}{n}$ for $n \geq 2$;

$$
C_{2}(\delta)=-\frac{i \pi}{\sin \delta / 2} \sum_{n=1}^{\infty}(-1)^{n} d(n) e^{-\pi n \cot \frac{\delta}{2}}
$$

and

$$
C_{3}(\delta)=\int_{0}^{\infty}|\zeta(1 / 2+i t)|^{2} \frac{-e^{-\pi t} \sinh t \delta+i \cosh t \delta}{\cosh \pi t} d t
$$


If $\delta$ is real this simplifies to

$$
\begin{aligned}
I(\delta)=-\frac{1+}{\log \left(2 \sin \frac{\delta}{2}\right)} & 2 \sin \frac{\delta}{2} \\
+ & \frac{1}{2 \sin \frac{\delta}{2}} \sum_{m=1}^{\infty} a_{m} \cos m \delta-\int_{0}^{\infty}|\zeta(1 / 2+i t)|^{2} \frac{e^{-\pi t} \sinh t \delta}{\cosh \pi t} d t
\end{aligned}
$$

We can use the fact that

$$
\sum_{m=1}^{\infty} a_{m}=\gamma+1-\log 2 \pi
$$

and that

$$
\frac{\cos m \delta-1}{2 \sin \frac{\delta}{2}}=-U_{m-1}\left(\cos \frac{\delta}{2}\right) \sin \frac{m \delta}{2}
$$

where $U_{m}$ is the $m$ th Chebyshev polynomial, to rewrite this further.

Corollary 1. - Suppose that $0<\delta<2 \pi$. Then

$$
\begin{aligned}
I(\delta)=\frac{\gamma-\log 2 \pi-\log \left(2 \sin \frac{\delta}{2}\right)}{2 \sin \frac{\delta}{2}}+(\pi-\delta) \cos \frac{\delta}{2}+(\log 2 \pi-\gamma) \sin \frac{\delta}{2} \\
-\sum_{m=1}^{\infty} a_{m} U_{m-1}\left(\cos \frac{\delta}{2}\right) \sin \frac{m \delta}{2}-\int_{0}^{\infty}|\zeta(1 / 2+i t)|^{2} \frac{e^{-\pi t} \sinh t \delta}{\cosh \pi t} d t
\end{aligned}
$$

In this version the first term above is the readily recognized usual main term. Note that the integral in the right-hand side of this formula can be rewritten in terms of the original integral $I$ :

$$
-\int_{0}^{\infty}|\zeta(1 / 2+i t)|^{2} \frac{e^{-\pi t} \sinh t \delta}{\cosh \pi t} d t=\sum_{n=1}^{\infty}(-1)^{n+1}(I(2 \pi n+\delta)-I(2 \pi n-\delta)) .
$$

Another way to put it is if we let

$$
w(\delta, t):=e^{-\delta t}+\frac{e^{-\pi t} \sinh \delta t}{\cosh \pi t}=\frac{\cosh (\pi-\delta) t}{\cosh \pi t},
$$

then

$$
\begin{gathered}
\int_{0}^{\infty}|\zeta(1 / 2+i t)|^{2} w(\delta, t) d t=\frac{\gamma-\log 2 \pi-\log \left(2 \sin \frac{\delta}{2}\right)}{2 \sin \frac{\delta}{2}}+\left(\frac{\pi}{2}-\delta\right) \cos \frac{\delta}{2}+(\log 2 \pi-\gamma) \sin \frac{\delta}{2} \\
+\sum_{m=1}^{\infty} a_{m} U_{m-1}\left(\cos \frac{\delta}{2}\right) \sin \frac{m \delta}{2}
\end{gathered}
$$

Since $a_{m} \ll e^{-\sqrt{m}}$ we have the following estimate for a precise evaluation of $I(\delta)$ as $\delta \rightarrow 0$.

Corollary 2. - Given $\delta>0$ and $N \geq 1$ we can compute $I(\delta)$ to an accuracy of $10^{-N}$ in time $t(\delta, N) \ll N^{2}$. 
This is fairly remarkable in that it doesn't depend on $\delta$ ! By contrast if one considers the associated integral

$$
\int_{0}^{\frac{1}{\delta}}|\zeta(1 / 2+i t)|^{2} d t
$$

then the time to calculate will depend on $\delta$ in a significant way, say $\delta^{-\theta}$ for some $\theta>0$.

Finally, using the fact that when $\delta$ is real, the imaginary part of $C_{3}(\delta)$ involves the weight $w(\pi-\delta, t)$ we deduce a formula for the divisor sum in $C_{2}(\delta)$ in terms of the coefficients $a_{m}$ :

Corollary 3. - For $0<\delta<\pi$

$$
\begin{gathered}
\frac{\pi}{\sin \frac{\delta}{2}} \sum_{n=1}^{\infty} d(n)(-1)^{n} e^{-n \pi \cot \frac{\delta}{2}}=-\frac{1}{\sin \delta} \sum_{m=1}^{\infty}\left(a_{2 m}+a_{2 m-1}\right) \sin \left(\left(2 m-\frac{1}{2}\right) \delta\right) \\
+\frac{\delta}{4 \sin \frac{\delta}{2}}-\frac{1+\log \left(2 \cos \frac{\delta}{2}\right)}{2 \cos \frac{\delta}{2}} .
\end{gathered}
$$

This can be rewritten as

$$
\begin{array}{r}
\sum_{m=1}^{\infty}\left(a_{2 m}+a_{2 m-1}\right) \sin \left(\left(2 m-\frac{1}{2}\right) \delta\right)=\frac{\delta \cos \frac{\delta}{2}}{2}-\sin \frac{\delta}{2}\left(1+\log \left(2 \cos \frac{\delta}{2}\right)\right) \\
-2 \pi \cos \frac{\delta}{2} \sum_{n=1}^{\infty} d(n)(-1)^{n} e^{-n \pi \cot \frac{\delta}{2}} .
\end{array}
$$

If $\delta=\frac{1}{1000}$, then the first term of the divisor sum on the right-hand side of the formula is $-1.12 \cdots \times 10^{-2729}$, whereas the first two terms on the right-hand side are $-0.000159155 \ldots$ That means that the sum on the left-hand side is $-0.000159155 \cdots-1.12 \cdots \times 10^{-2729}$ up to an error of around $-1.99 \cdots \times 10^{-5458}$, which is the second term of the divisor sum. Since the terms $a_{2 m}$ are around $e^{-2 \sqrt{2 \pi m}}$ it would take more than 6 million terms of the series in $m$ (each with thousands of digits of accuracy) to numerically check this.

\section{Proof of Theorem 1}

Assume first of all that $\delta$ is real with $0<\delta<\pi$. We have

$$
I(\delta)=\frac{1}{i} \int_{1 / 2}^{1 / 2+i \infty} \zeta(s) \zeta(1-s) e^{i \delta(s-1 / 2)} d s=\frac{e^{-i \delta / 2}}{i} \int_{1 / 2}^{1 / 2+i \infty} \chi(1-s) \zeta(s)^{2} e^{i \delta s} d s .
$$

Now

$$
\chi(1-s)=(2 \pi)^{-s} \Gamma(s)\left(e^{\pi i s / 2}+e^{-\pi i s / 2}\right)
$$

so that

$$
I(\delta)=I_{1}(\delta)+I_{2}(\delta)
$$

where

$$
I_{1}(\delta)=\frac{e^{-i \delta / 2}}{i} \int_{1 / 2}^{1 / 2+i \infty}(2 \pi)^{-s} \Gamma(s) e^{-\pi i s / 2} \zeta(s)^{2} e^{i \delta s} d s
$$


and

$$
I_{2}(\delta)=\frac{e^{-i \delta / 2}}{i} \int_{1 / 2}^{1 / 2+i \infty}(2 \pi)^{-s} \Gamma(s) e^{\pi i s / 2} \zeta(s)^{2} e^{i \delta s} d s
$$

Now

$$
I_{1}(\delta)=I_{0}(\delta)-I_{3}(\delta)
$$

where

$$
I_{0}(\delta)=\frac{e^{-i \delta / 2}}{i} \int_{1 / 2-i \infty}^{1 / 2+i \infty}(2 \pi)^{-s} \Gamma(s) e^{-\pi i s / 2} \zeta(s)^{2} e^{i \delta s} d s
$$

and

$$
I_{3}(\delta)=\frac{e^{-i \delta / 2}}{i} \int_{1 / 2-i \infty}^{1 / 2}(2 \pi)^{-s} \Gamma(s) e^{-\pi i s / 2} \zeta(s)^{2} e^{i \delta s} d s .
$$

Thus, $I(\delta)=I_{0}(\delta)+I_{2}(\delta)-I_{3}(\delta)$. Note that $I_{2}(\delta)$ and $I_{3}(\delta)$ are analytic for $|\delta|<\pi / 2$. We rewrite $I_{2}$ and $I_{3}$ as integrals over $t$ as

$$
\begin{aligned}
I_{2}(\delta) & =\frac{e^{-i \delta / 2}}{i} \int_{1 / 2}^{1 / 2+i \infty} \zeta(s) \zeta(1-s) \frac{e^{\pi i s / 2}}{2 \cos \pi s / 2} e^{i \delta s} d s \\
& =\int_{0}^{\infty}|\zeta(1 / 2+i t)|^{2} e^{-\pi t / 2} \frac{e^{\pi i / 4} e^{-\delta t}}{2 \cos \frac{\pi}{2}(1 / 2+i t)} d t
\end{aligned}
$$

and

$$
\begin{aligned}
I_{3}(\delta) & =\frac{e^{-i \delta / 2}}{i} \int_{1 / 2-i \infty}^{1 / 2} \zeta(s) \zeta(1-s) \frac{e^{-\pi i s / 2}}{2 \cos \pi s / 2} e^{i \delta s} d s \\
& =\frac{e^{-i \delta / 2}}{i} \int_{1 / 2}^{1 / 2+i \infty} \zeta(s) \zeta(1-s) \frac{e^{-\pi i(1-s) / 2}}{2 \cos \pi(1-s) / 2} e^{i \delta(1-s)} d s \\
& =\int_{0}^{\infty}|\zeta(1 / 2+i t)|^{2} e^{-\pi t / 2} \frac{e^{-\pi i / 4} e^{\delta t}}{2 \cos \frac{\pi}{2}(1 / 2-i t)} d t
\end{aligned}
$$

Next we write $e^{\delta t}=\cosh \delta t+\sinh \delta t$ and $e^{-\delta t}=\cosh \delta t-\sinh \delta t$. Also, for real $t$,

$$
\frac{e^{\pi i / 4}}{2 \cos \frac{\pi}{2}(1 / 2+i t)}+\frac{e^{-\pi i / 4}}{2 \cos \frac{\pi}{2}(1 / 2-i t)}=\frac{e^{-\pi t / 2}}{\cosh \pi t}
$$

and

$$
\frac{e^{\pi i / 4}}{2 \cos \frac{\pi}{2}(1 / 2+i t)}-\frac{e^{-\pi i / 4}}{2 \cos \frac{\pi}{2}(1 / 2-i t)}=i \frac{e^{\pi t / 2}}{\cosh \pi t}
$$

Thus,

$$
I_{2}(\delta)-I_{3}(\delta)=\int_{0}^{\infty}|\zeta(1 / 2+i t)|^{2} \frac{-e^{-\pi t} \sinh t \delta+i \cosh t \delta}{\cosh \pi t} d t
$$

Returning to $I_{0}$ we have

$$
\begin{aligned}
I_{0}(\delta) & =-2 \pi e^{-i \delta / 2} \operatorname{Res}_{s=1}(2 \pi)^{-s} \Gamma(s) e^{-\pi i s / 2} \zeta(s)^{2} e^{i \delta s}+J(\delta) \\
& =e^{i \delta / 2}(-\delta+\pi / 2+i \gamma-i \log 2 \pi)+J(\delta)
\end{aligned}
$$


where

$$
J(\delta)=\frac{e^{-i \delta / 2}}{i} \int_{2-i \infty}^{2+i \infty}(2 \pi)^{-s} \Gamma(s) e^{-\pi i s / 2} \zeta(s)^{2} e^{i \delta s} d s
$$

note that this is a place where we need the (temporary) assumption that $\delta$ is real to ensure convergence of the integral on the new path. Expanding $\zeta(s)^{2}=\sum_{n=1}^{\infty} d(n) n^{-s}$ into its Dirichlet series and interchanging the summation and integration, we obtain

$$
\begin{aligned}
J(\delta) & =2 \pi e^{-i \delta / 2} \sum_{n=1} d(n) \frac{1}{2 \pi i} \int_{2-i \infty}^{2+i \infty} \Gamma(s)\left(2 \pi i n e^{-i \delta}\right)^{-s} d s \\
& =2 \pi e^{-i \delta / 2} \sum_{n=1}^{\infty} d(n) e^{-2 \pi i n e^{-i \delta}} \\
& =\frac{\pi}{2} e^{-i \delta / 2}\left(1-E_{1}\left(-e^{-i \delta}\right)\right) .
\end{aligned}
$$

Now

$$
E_{1}\left(-e^{-i \delta}\right)=E_{1}\left(1-e^{-i \delta}\right)=\frac{1}{1-e^{-i \delta}} E_{1}\left(\frac{-1}{1-e^{-i \delta}}\right)+\psi\left(1-e^{-i \delta}\right)
$$

and this is

$$
\frac{1}{1-e^{-i \delta}}\left(1-4 \sum_{n=1}^{\infty} d(n) e\left(\frac{-n}{1-e^{-i \delta}}\right)\right)+\psi\left(1-e^{-i \delta}\right)
$$

Thus,

$$
\begin{aligned}
J(\delta) & =\frac{\pi}{2} e^{-i \delta / 2}-\frac{\frac{\pi}{2} e^{-i \delta / 2}}{1-e^{-i \delta}}\left(1-4 \sum_{n=1}^{\infty} d(n) e\left(\frac{-n}{1-e^{-i \delta}}\right)\right)-\frac{\pi}{2} e^{-i \delta / 2} \psi\left(1-e^{-i \delta}\right) \\
& =\frac{\frac{\pi}{2} e^{-i \delta / 2}}{1-e^{i \delta}}-\frac{i \pi}{\sin \delta / 2} \sum_{n=1}^{\infty}(-1)^{n} d(n) e^{-\pi n \cot \frac{\delta}{2}}-\frac{\pi}{2} e^{-i \delta / 2} \psi\left(1-e^{-i \delta}\right) .
\end{aligned}
$$

Altogether we now have

$$
\begin{aligned}
\int_{0}^{\infty}|\zeta(1 / 2+i t)|^{2} e^{-\delta t} d t=\frac{\frac{\pi}{2} e^{-i \delta / 2}}{1-e^{i \delta}} & +e^{i \delta / 2}(-\delta+\pi / 2+i \gamma-i \log 2 \pi) \\
& -\frac{\pi}{2} e^{-i \delta / 2} \psi\left(1-e^{-i \delta}\right) \\
& -\frac{i \pi}{\sin \delta / 2} \sum_{n=1}^{\infty}(-1)^{n} d(n) e^{-\pi n \cot \frac{\delta}{2}} \\
& +\int_{0}^{\infty}|\zeta(1 / 2+i t)|^{2} \frac{-e^{-\pi t} \sinh t \delta+i \cosh t \delta}{\cosh \pi t} d t
\end{aligned}
$$

Recall that

$$
\psi(z)=-\frac{2(\log z+1)}{\pi i z}+\frac{2}{\pi i z} \sum_{m=1}^{\infty} a_{m}(-1)^{m}(z-1)^{m}
$$


so that

$$
\psi\left(1-e^{-i \delta}\right)=-\frac{2\left(\log \left(1-e^{-i \delta}\right)+1\right)}{\pi i\left(1-e^{-i \delta}\right)}+\frac{2}{\pi i\left(1-e^{-i \delta}\right)} \sum_{m=1}^{\infty} a_{m} e^{-i m \delta}
$$

and

$$
-\frac{\pi}{2} e^{-i \delta / 2} \psi\left(1-e^{-i \delta}\right)=-\frac{\left(\log \left(1-e^{-i \delta}\right)+1\right)}{2 \sin \frac{\delta}{2}}+\frac{1}{2 \sin \frac{\delta}{2}} \sum_{m=1}^{\infty} a_{m} e^{-i m \delta} .
$$

The assertion of the theorem now follows for real $\delta$. But both sides are analytic in the region $0<\Re<\pi$ and $\Im \delta<0$. Therefore, by analytic continuation the identity of the theorem holds in this larger region of the complex plane.

\section{References}

[BC] Bettin, Sandro; Conrey, Brian, Period functions and cotangent sums. Algebra Number Theory 7 (2013), no. 1, 215-242.

16 aout 2013

S. Bettin, Centre de Recherches Mathematiques Universite de Montreal, P. O Box 6128, CentreVille Station, Montreal, Quebec H3C 3J7 • E-mail : bettin@crm.umontreal.ca

J.B. Conrey, American Institute of Mathematics, 360 Portage Ave, Palo Alto, CA 94306 USA and School of Mathematics, University of Bristol, Bristol, BS8 1TW, United Kingdom

E-mail : conrey@aimath.org 